\title{
On the mathematical form of psychophysical relationships, with special focus on the perception of mechanical properties of solid objects
}

\author{
M. PELEG and O. H. CAMPANELLA \\ University of Massachusetts, Amherst, Massachusetts
}

\begin{abstract}
Because the sensory response to mechanical and other stimuli cannot increase indefinitely, the power law of sensory perception can be valid only within a limited range of stimulus magnitudes. An alternative two-parameter mathematical expression that can describe, at least in principle, psychophysical relationships at any stimulus range is presented. It is shown mathematically, and demonstrated through computer simulation, that unless the stimulus magnitude is too close to the threshold or saturation level, the resulting relationship in logarithmic coordinates is practically identical to that predicted by the power law model. It is also demonstrated that the absolute magnitude of the alternative model's constants can be very similar to those found for the power law model.
\end{abstract}

Psychophysical relationships have commonly been presented in the form of a power relationship between the sensation magnitude $(\psi)$ and the stimulus intensity $(\phi)$ (Gescheider, 1985; Marks, 1974; Stevens, 1975), or

$$
\psi=K \phi^{n},
$$

where $n$ is a coefficient, the magnitude of which is characteristic of the type of stimulus. Values of $n$ reported by Stevens (1975) range from 3.5 for electric shock to 0.33 for brightness detection. Assessments of mechanical properties such as muscle force, heaviness, or hardness are usually on the order of 0.4-2 (Harper \& Stevens, 1964; Moskowitz, 1977; Moskowitz, Segars, Kapsalis, \& Kluter, 1974; Stevens, 1975).

Equation 1 is frequently transformed to a logarithmic relationship, that is,

$$
\log \psi=K^{\prime}+n \log \phi
$$

where $K^{\prime}=\log K$. Since for many stimuli there is a threshold level $\left(\phi_{0}\right)$, Equation 1 can also be presented in the form

$$
\psi=K\left(\phi-\phi_{0}\right)^{n},
$$

or its logarithmic equivalent

$$
\log \psi=K^{\prime}+n \log \left(\phi-\phi_{0}\right)
$$

This work was part of a project supported by the Biochemical and Biomass Engineering Program of the National Science Foundation (Grant CBT 8702877). The authors express their thanks to the NSF for the support and to Richard J. Grant for the graphical aid. The authors also want to thank Kenneth Norwich for his suggestion to include Equations 7-10 and their discussion in the text. Address reprint requests to $M$. Peleg, Department of Food Engineering, University of Massachusetts, Amherst, MA 01003.
The mathematical simplicity and convenience of the relationships expressed by Equations 1-4 are undeniable, and it appears that there is ample experimental evidence to support their validity. Certain theoretical aspects of the relationships, particularly those that are associated with the definition of $\psi$ and the methodology of its determination, have been a topic of ongoing study and discussion (e.g., Falmagne, 1985; Gescheider, 1985; Shepard, 1981).

As far as the perception of mechanical attributes is concerned, the construction of a psychophysical relationship is associated with a few unresolved problems. One, of a semantic nature, originates from the ambiguity and overlap in the meaning of terms such as hardness, firmness, and toughness, and their relation to different mechanical stimuli (Peleg, 1983a). Thus, resistance to deformation and difficulty in sustaining permanent indentation, although defined as "hardness," are really two different mechanical properties that can vary independently among materials. Another problem is the difficulty in determining the true magnitude of the stimulus because of the mechanical interaction between the assessed object and the soft viscoelastic tissues through which it is sensed (Peleg, 1983b). This difficulty is further aggravated because the tissues undergo stress relaxation or creep. These and the viscoelasticity of the mechanoreceptors themselves would cause the stimulus to decay or vary in time independently of the phenomenon known as adaptation, which is primarily caused by electrochemical processes within the mechanoreceptors (Loewenstein, 1971).

Let us assume, however, that a meaningful, or at least workable, relationship between a mechanical stimulus and its perceived intensity can be derived. If such a relationship were in the form of Equation 1 or Equation 3 (Figure 1a), it would imply that the response intensity can 
(a)

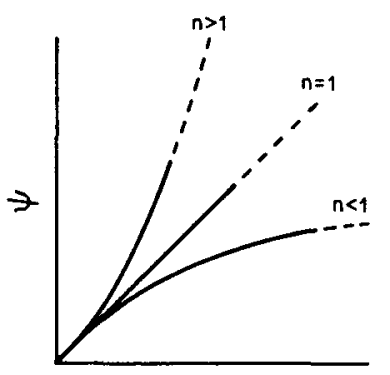

(b)

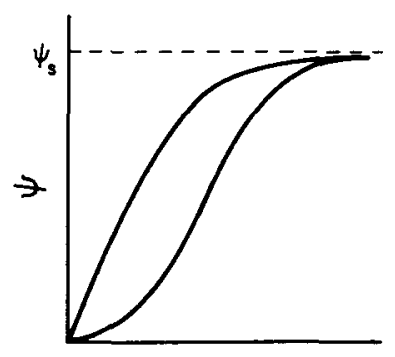

(c)

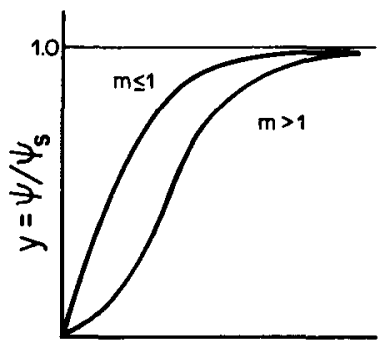

$$
x=\phi \text { or } \phi-\phi_{0}
$$

Figure 1. Schematic presentation of the types of psychophysical relationships. $\phi$ is the stimulus intensity, $\phi_{0}$ the threshold stimulus, $\psi$ the response intensity, and $\psi_{s}$ the saturation level of the response. (The scales are arbitrary.)

increase indefinitely. This, of course, is physically impossible. In reality, the response intensity ought to stabilize at a level dictated by the saturation of the mechanoreceptors; exceeding this level may result in pain. In other words, if the whole range of possible stimuli is taken into account, the general shape of the relationship is most likely one of the kinds shown in Figure 1b, that is, exhibiting an asymptotic response level. The fact that the sensory response cannot increase indefinitely in hardness evaluation has already been recognized by Harper and Stevens (1964). They introduced the term upper threshold to describe the stimulus level at which the sensory response stops increasing with the stimulus. They also proposed a mathematical expression to account for the existence of both upper and lower thresholds. This expression, however, has a singularity at the upper threshold level and not an asymptotically reached constant response. Their model, therefore, is probably appropriate for the description of the conditions for pain inception, but not for describing the phenomenon of response stabilization.

The objectives of this communication are to demonstrate a simple alternative mathematical model that can describe psychophysical relationships that have an asymptotic response level, to compare its properties with those of the conventional psychophysical model, and to explore some of its implications and consequences in sensory evaluations of mechanical and other attributes.

\section{The General Psychophysical Relationships}

Let us assume that, irrespective of the scale used for its quantification, the sensory response $(\psi)$ must approach asymptotically a saturation level $\left(\psi_{s}\right)$. In such a case, the response can be normalized in the form of

$$
Y=\frac{\psi}{\psi_{s}},
$$

where $Y$ is the normalized, dimensionless response that can vary between zero and unity. If the shape of $Y$ versus $\phi$ or $Y$ versus $\phi-\phi_{0}$ is of the general form shown in Figure 1c, it can be described or at least be approximated by a variety of functions. Let us, however, examine the properties of only one mathematical expression, of the kind

$$
Y=1-\exp \left(-C x^{m}\right),
$$

where $x=\phi$ or $\phi-\phi_{0}$, and $C$ and $m$ are constants.

Demonstration of the features of Equation 6 are shown in Figures 2 and 3. In Figure 2 the value of $C$ was kept fixed and that of $m$ was allowed to vary, whereas in Figure $3 C$ varied and $m$ was fixed. It ought to be noticed that when $m<1$, irrespective of the magnitude of $C$, the sigmoid shape of the curve is lost and the slope progressively decreases. Such curves are most probably fit only for relationships beyond the threshold, that is, where $x=\phi-\phi_{0}$.

Comparison between Equation 6 and conventional psychophysical relationships. Most experimental psychophysical relationships are derived from the linear

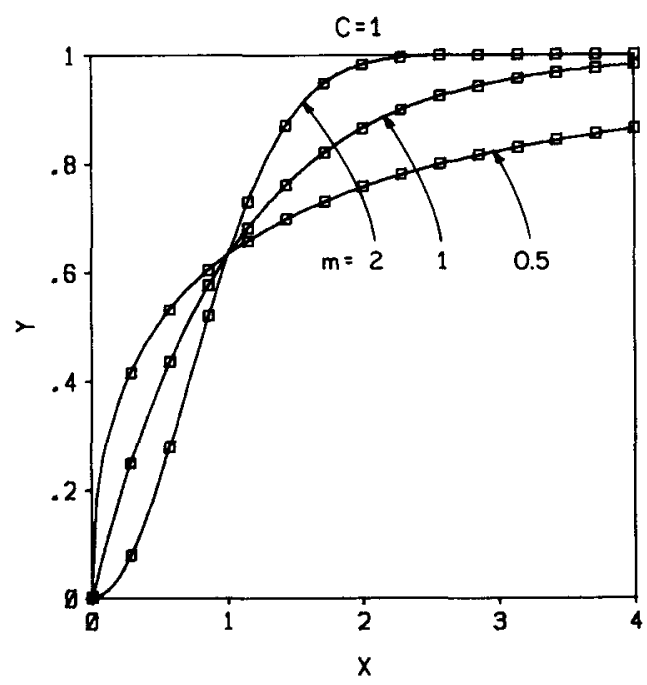

Figure 2. Examples of normalized response intensity $(Y)$ versus effective stimulus $(x)$ generated by a computer using Equation 6 (constant $C$ and variable $m$ ). 


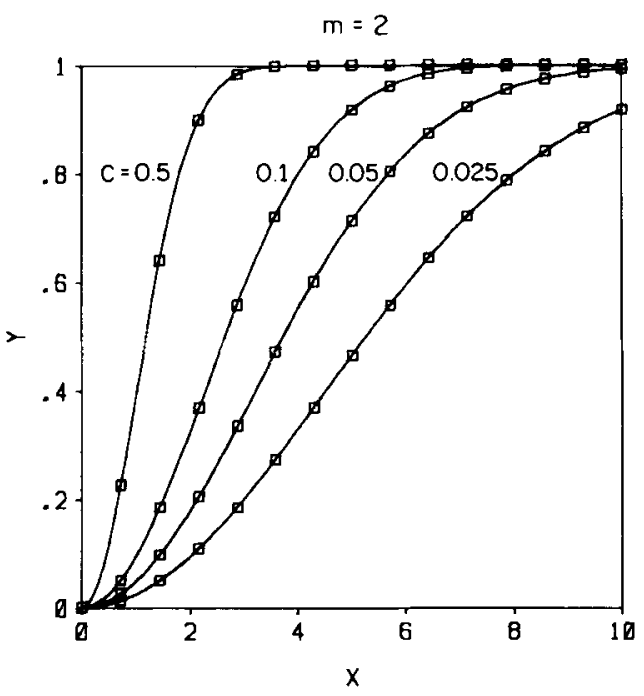

Figure 3. Examples of normalized response intensity $(Y)$ versus effective stimulus $(x)$ generated by a computer using Equation 6 (constant $m$ and variable $C$ ).

relationsips of $\log \psi$ versus $\log \phi$ or $\log \left(\phi-\phi_{0}\right)$, which are used to calculate the values of $K$ and $n$. It can be shown that considerable portions of the relationships expressed by Equation 6 will also appear linear in a log-log plot, provided that the "saturation" region is not reached. Demonstrations of such linear regions are given in Figures 4 and 5 . Obviously, the actual range of the linear region depends on the constants $C$ and $m$. The point, however, is that a region covering two or more orders of magnitude of $x$ is a common feature of such plots, and therefore the model expression in Equation 6 is not incompatible with the conventional models presented by Equations 1-4.

The linearity of the $\log -\log$ plot of data generated by Equation 6 over a wide range of stimulus intensity is not a mathematical coincidence. It can be shown that the linear relationship can be derived directly from Equation 6 in the following manner.

The Maclaurin series expansion of $e^{z}$ is

$$
e^{Z}=1+Z+\frac{Z^{2}}{2 !}+\frac{Z^{3}}{3 !}+\ldots
$$

For positive values of $x$ and $Y(x)$, one can substitute $-C x^{m}$ for $Z$ to yield

$$
e^{-C x^{m}}=1-C x^{m}+\frac{C^{2} x^{2 m}}{2 !}-\frac{C^{3} x^{3 m}}{3 !}+\ldots
$$

Consequently, the values of $Y(x)$, as defined by Equation 6 , can be calculated from the series

$$
Y(x)=1-e^{-C x^{m}}=C x^{m}-\frac{C^{2} x^{2 m}}{2 !}+\frac{C^{3} x^{3 m}}{3 !}-\ldots
$$

For small values of $x$, the sum of the second and other terms of the right side of the equation becomes negligi- ble in comparison with the magnitude of the first term, and therefore it can be dropped. In mathematical terms, this can be done whenever

$$
x^{m} \ll \frac{2}{C} \text {. }
$$

Under these circumstances Equation 9 is rendered

$$
Y(x)=C x^{m},
$$

which is identical to the original power law equation with $m=n$ and $C=K \psi_{s}$. By the same reasoning, the inception of clear curvature in the $\log Y$ versus $\log x$ plot will occur at stimulus levels for which the condition expressed by Equation 10 is not satisfied.

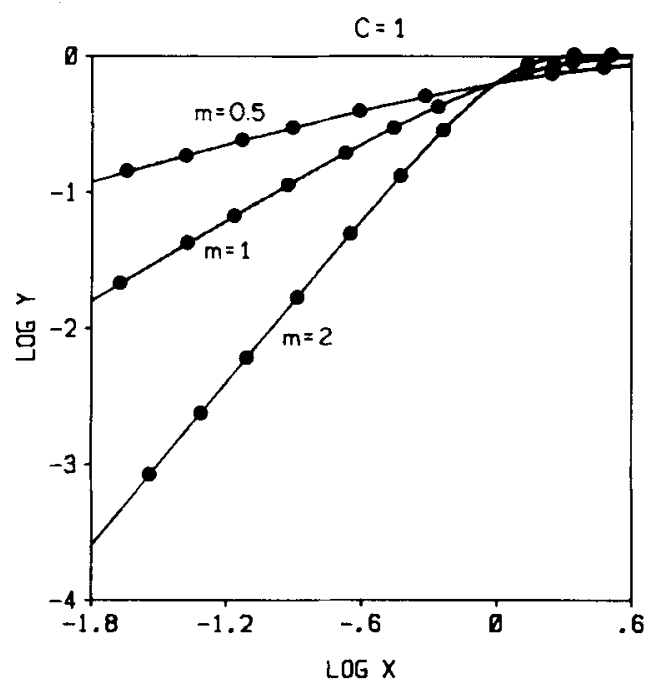

Figure 4. The data of Figure 2 presented as logarithmic relationships. Note the range of the linear regions. (For the regression analysis and more details, see Table 1.)

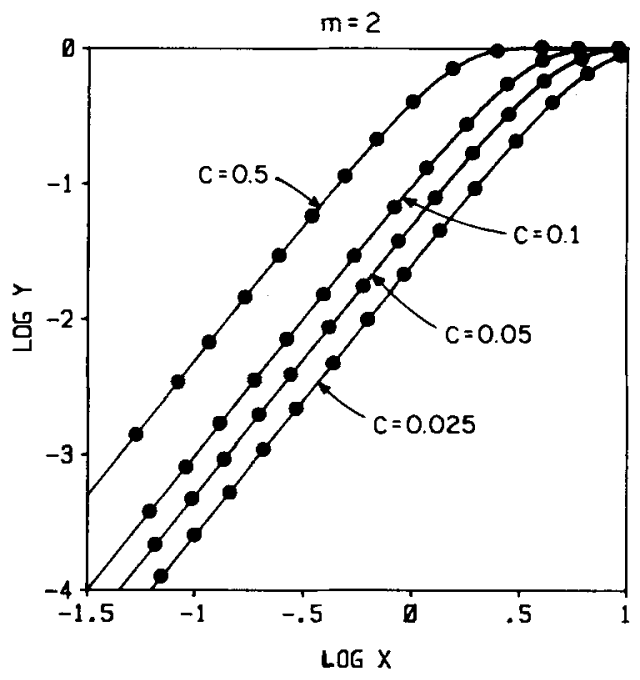

Figure 5. The data of Figure 3 presented as logarithmic relationships. Note the range of the linear regions. (For the regression analysis and more details, see Table 1.) 
It should also be noted that the relationships shown in Figures 2-5 were generated by a computer and therefore represent mathematical relationships. Experimentally determined relationships are rarely as smooth, and considerable data scatter is quite common (e.g., Moskowitz et al., 1974). Because of the latter, detection of the true curvature of the $\log \psi$ versus $\log \phi$ or $\log \left(\phi-\phi_{0}\right)$ relationship is not always easy, even when the stimulus magnitude is fairly close to the saturation level. Furthermore, it can be shown from basic mechanical principles that the sensory sensitivity considerably decreases, and almost completely vanishes, when the object hardness is the same as or greater than that of body tissues that are in contact with it (Norwich, 1972). The reason for this is that the softer the tissues are relative to the asessed object, the greater is the part of the deformation that is sustained by the tissues (Peleg, 1980a, 1980b). In the extreme case, as when we press a metal object between the fingers, virtually all the deformation is in the fingers themselves. This corresponds to testing the fingers rather than the metal object, a situation that is equivalent to zero mechanical sensitivity with respect to the metal object. In these and similar cases, a large scatter in the data in the regions close to the asymptotic level is not a reflection of random error but an inevitable outcome of the sensation mechanism itself.

\section{Relationship Between the Proposed and the Power Law Model Constants}

To demonstrate the characteristics of the proposed model (Equation 6), the central regions of $\log Y$ versus $\log x$ plots were subjected to linear regression to calculate the slope and intercept. The plots were generated with the constant $m$ in the range of $0.5-2$, corresponding to the common range of the power in psychophysical relationships (see below). The magnitude of $C$ was arbitrarily selected to cover different ranges of $x$. Since $x$ is expressed in the units of the objective stimulus, it can, in

Table 1

Regression Parameters and Fit of the Logarithmic Psychophysical Law (Equation 2 or 4) to Data Generated by Equation $6^{*}$

\begin{tabular}{lcccccc}
\hline \multicolumn{1}{c}{$\begin{array}{c}K \\
C\end{array}$} & (Fitted) & $m$ & $\begin{array}{c}n \\
\text { (Fitted) }\end{array}$ & $\begin{array}{c}\text { Range } \\
\text { of } x\end{array}$ & $\begin{array}{c}\text { No. of } \\
\text { Points }\end{array}$ & $r^{2}$ \\
\hline 0.001 & 0.001 & 0.5 & 0.50 & $0.01-50$ & 121 & 0.9999 \\
0.001 & 0.001 & 1.0 & 1.00 & $0.01-50$ & 121 & 0.9999 \\
0.001 & 0.001 & 2.0 & 1.91 & $0.01-50$ & 121 & 0.9983 \\
0.01 & 0.01 & 0.5 & 0.50 & $0.01-10$ & 82 & 0.9999 \\
0.01 & 0.01 & 1.0 & 0.99 & $0.01-10$ & 82 & 0.9999 \\
0.01 & 0.009 & 2.0 & 1.96 & $0.01-10$ & 82 & 0.9992 \\
0.1 & 0.094 & 0.5 & 0.48 & $0.01-10$ & 83 & 0.9995 \\
0.1 & 0.092 & 1.0 & 0.96 & $0.01-8$ & 79 & 0.9986 \\
0.1 & 0.093 & 2.0 & 1.96 & $0.01-3$ & 74 & 0.9995 \\
1.0 & 0.68 & 0.5 & 0.41 & $0.01-0.8$ & 61 & 0.9968 \\
1.0 & 0.76 & 1.0 & 0.91 & $0.01-0.8$ & 61 & 0.9979 \\
1.0 & 0.87 & 2.0 & 1.95 & $0.01-0.8$ & 61 & 0.9995 \\
2.0 & 1.00 & 0.5 & 0.35 & $0.01-0.5$ & 50 & 0.9899 \\
2.0 & 1.26 & 1.0 & 0.86 & $0.01-0.5$ & 50 & 0.9952 \\
2.0 & 1.65 & 2.0 & 1.93 & $0.001-0.5$ & 50 & 0.9993 \\
\hline *See also Figures $2-5$. & & & &
\end{tabular}

*See also Figures 2-5. theory, assume any level. Typical results are shown in Table 1. As could be expected, the table shows that the values of $m$ in Equation 6 roughly correspond to the values of $n$ in the conventional power law models when the magnitude of $C$ is relatively high and are practically identical with the value of $n$ when the magnitude of $C$ is small. The table also demonstrates that, if similar data, especially with some scatter, are subjected to linear regression, a relationship of the kind expressed by Equations 2 or 4 will naturally emerge with the true curvature probably masked by the scatter. In other words, if the true relationship between $\psi$ and $x$ obeys, over the whole range, an equation of the kind expressed by Equation 6, there always will be ranges of stimulus intensity for which the conventional model will be found valid within an acceptable level of statistical confidence.

\section{Implications in Sensory Analyses}

In most sensory analyses, the stimulus intensity is in the intermediate range, that is, far enough from both the lower threshold and the saturation level. Notable exceptions are those experiments designed especially to establish the threshold or pain levels, or to determine the just noticeable difference versus stimulus intensity relationships, but they should not concern us here. In the intermediate range, as previously mentioned, psychophysical relationships of the kind described by Equations 1-4 will most likely emerge with high statistical confidence level. Since the validity of these relationships is frequently taken for granted, the pertinent range of the particular stimulus is rarely determined prior to the test. Therefore, there is a danger, particularly when hard objects (e.g., hard candy, biscuits) are sensorily evaluated, that the stimulus range is already in the near saturation level. Since the human subjects must report their evaluations, the latter, together with their considerable scatter (that results from a sensitivity loss), will enter the calculation and will lower the determined value of the constant $n$. This scenario is probably not uncommon with other types of stimuli for which the saturation range can be easily reached. It is, therefore, advisable in such cases to determine a priori the pertinent saturation level so that the results are not distorted. When the true relationship is of a sigmoid shape, similar problems can arise at the other end of the scale. In such cases, and again as a result of sensitivity loss and data scatter, an overestimate of the parameter $n$ will most likely be reached.

It should be stated again that the model expressed in Equation 6 need not be a unique representation of psychophysical relationships, irrespective of whether the latter refer to hardness or any other attribute. It was primarily selected for its mathematical simplicity and for having two constants only; that is, it is not much more complicated than the conventional model. Theoretically, however, there is no reason why the same mathematical relationship ought to be valid throughout the entire range, or that a comprehensive model must have only two constants. It is, therefore, quite possible that more elaborate models 
will be found more appropriate for the entire range. Determining the constants of this model, or possibly of the more elaborate ones, does not pose any serious problem. Since nonlinear regression has become almost a standard item of computer software, the loss of convenience that stems from dealing with linear $\log$-log relationships is no longer a real drawback as far as the ease of computation is concerned.

\section{REFERENCES}

FALMAGNE, J. C. (1985). Elements of psychophysical theory. New York: Oxford University Press.

Gescheider, G. A. (1985). Psychophysics: Method, theory and application. Hillsdale, NJ: Erlbaum.

HARPER, R., STEvens, S. S. (1964). Subjective hardness of compliant materials. Quarterly Joumal of Experimental Psychology, 16, 204215.

Loewenstein, W. R. (1971). Principles of receptor physiology. Berlin: Springer-Verlag.

MARKs, L. E. (1974). Sensory processes-The new psychophysics. New York: Academic Press.

Moskowrtz, H. R. (1977). Correlating sensory and instrumental measures in food texture. Cereal Food World, 22, 232-237.
Moskowitz, H. R., Segars, R. A., Kapsalus, J. G., Kluter, R. A. (1974). Sensory ratio scales relating hardness and crunchiness to mechanical properties of space cubes. Journal of Food Science, 39, 200-202.

NoRWICH, K. H. (1972). The tactile discrimination of complex systems. International Journal of Systems Science, 2, 225-228.

PELEG, M. (1980a). A note on the sensitivity of fingers, tongue and jaws as mechanical testing instruments. Journal of Texture Studies, $10,245-251$.

Peleg, M. (1980b). Theoretical analysis of the relationship between mechanical hardness and its sensory assessment. Journal of Food Science, 45, 1156-1160.

Peleg, M. (1983a). The semantic of rheology and texture. Food Technology, 37, 54-61.

PELEG, M. (1983b). Some theoretical characteristics of the mechanical signals in sensory evaluation of texture. Journal of Food Science, 48, 1187-1191.

SHEPARD, R. N. (1981). Psychological relations and psychophysical scales: On the status of "direct" psychophysical measurement. Joumal of Mathematical Psychology, 24, 21-57.

Stevens, S. S. (1975). Psychophysics: Introduction to its perceptual, neural and social prospects. New York: Wiley.

(Manuscript received October 26, 1987; revision accepted for publication May 11, 1988.) 